EPJ manuscript No.

(will be inserted by the editor)

\title{
Calculation of photoemission spectra of the doped Mott insulator $\mathrm{La}_{1-x} \mathrm{Sr}_{x} \mathrm{TiO}_{3}$ using LDA+DMFT(QMC)
}

\author{
I.A. Nekrasov ${ }^{1, a}$, K. Held ${ }^{2, b}$, N. Blümer ${ }^{2}$, A.I. Poteryaev ${ }^{1}$, V.I. Anisimov ${ }^{1}$, and D. Vollhardt ${ }^{2}$ \\ ' Institute of Metal Physics, Russian Academy of Sciences-Ural Division, \\ 620219 Yekaterinburg GSP-170, Russia \\ ${ }^{2}$ Theoretische Physik III, Elektronische Korrelationen und Magnetismus, \\ Universität Augsburg, D-86135 Augsburg, Germany
}

October 25, 2018

\begin{abstract}
The spectral properties of $\mathrm{La}_{1-x} \mathrm{Sr}_{x} \mathrm{TiO}_{3}$, a doped Mott insulator with strong Coulomb correlations, are calculated with the ab initio computational scheme LDA+DMFT(QMC). It starts from the non-interacting electronic band structure as calculated by the local density approximation (LDA), and introduces the missing correlations by the dynamical mean-field theory (DMFT), using numerically exact quantum Monte-Carlo (QMC) techniques to solve the resulting self-consistent multi-band single-impurity problem. The results of the LDA+DMFT(QMC) approach for the photoemission spectra of $\mathrm{La}_{1-x} \mathrm{Sr}_{x} \mathrm{TiO}_{3}$ are in good agreement with experiment and represent a considerable qualitative and quantitative improvement on standard LDA calculations.
\end{abstract}

PACS. 71.27.+a Strongly correlated electron systems; heavy fermions - 74.25.Jb Electronic structure 79.60.-i Photoemission and photoelectron spectra

\section{Introduction}

At present, the electronic properties of solids are investigated by two essentially separate communities, one using model Hamiltonians in conjunction with many-body techniques, the other employing density functional theory (DFT) [1]. DFT and its local density approximation (LDA) have the advantage of being ab initio approaches which do not require empirical parameters as input. Indeed, they are highly successful techniques for the calculation of the electronic structure of real materials [2]. However, in practice DFT/LDA is seriously restricted in its ability to describe strongly correlated materials where the on-site Coulomb interaction is comparable with the band width. Here, the model Hamiltonian approach is more general and powerful since there exist systematic theoretical techniques to investigate the many-electron problem with increasing accuracy. Nevertheless, the uncertainty in the choice of the model parameters and the technical complexity of the correlation problem itself prevent the model Hamiltonian approach from being a flexible or reliable enough tool for studying real materials. The two approaches are therefore complementary. In view of the individual power of DFT/LDA and the model Hamiltonian approach, respectively, it would be highly desirable to be able to combine these techniques, thereby creating

\footnotetext{
a e-mail: nekrasov@ifmlrs.uran.ru

b e-mail: held@physik.uni-augsburg.de
}

an enormous potential for future $a b$ initio investigations of all real materials, including, e.g., $f$-electron systems and Mott insulators. One of the first successful attempts in this direction was the LDA+U method [3], which combines LDA with a basically static, i.e., Hartree-Fock-like, mean-field approximation for a multi-band Anderson lattice model (with interacting and non-interacting orbitals). This method proved to be a very useful tool in the study of long-range ordered, insulating states of transition metals and rare-earth compounds. However, the paramagnetic metallic phase of correlated electron systems such as hightemperature superconductors and heavy-fermion systems clearly requires a treatment that goes beyond a static mean-field approximation and includes dynamical effects, e.g., the frequency dependence of the self-energy.

During the last decade a new many-body approach was developed which is especially well-suited for the investigation of strongly correlated metals - the dynamical mean-field theory (DMFT) [4,5, 6]. It becomes exact in the limit of high lattice coordination numbers [7] and preserves the dynamics of local interactions; hence it represents a dynamic mean-field approximation. In this nonperturbative approach, the lattice problem is mapped onto an effective Anderson impurity model with a hybridization function which has to be determined self-consistently. To solve the effective impurity problem one can either use approximative techniques such as iterated perturbation theory (IPT) 8, 6] and the non-crossing approxima- 
tion (NCA) 9, 10, 11], or employ numerical techniques like quantum Monte-Carlo simulations (QMC) 12], exact diagonalization (ED) [13, 6], and numerical renormalization group (NRG) 14. In principle, QMC, ED and NRG are exact methods, but require an extrapolation: discretization of the imaginary time $\Delta \tau \rightarrow 0$ (QMC), the number of lattice sites of the respective impurity model $n_{s} \rightarrow \infty$ (ED), and the parameter for logarithmic discretization of the conducting band $\Lambda \rightarrow 1$ (NRG), respectively.

In principle, the main idea of the $\mathrm{LDA}+\mathrm{U}$ method [3] (i.e., complementing the LDA band structure by a screened Coulomb interaction between localized tight-binding orbitals) can be practically applied with more refined approximation schemes 15, 16, 17, 18, 19, 20]. Indeed, a calculation scheme supplementing LDA with DMFT to include dynamic effects was first formulated by Anisimov et al. [15] and was used to calculate the photoemission spectra of $\mathrm{La}_{1-x} \mathrm{Sr}_{x} \mathrm{TiO}_{3}$, a doped Mott-insulator and strongly correlated paramagnetic metal, in connection with IPT [15] and NCA 20. The LDA++ approach by Lichtenstein and Katsnelson 16 formulates a very similar strategy and was recently applied to investigate correlation effects in iron 21,22]. Both IPT and NCA are approximative methods to solve the effective single-impurity problem in the LDA+DMFT scheme. They have the advantage of being numerically inexpensive, but their reliability, especially in the case of multi-band systems with particle densities off half-filling, is in principle uncertain. In this situation, it is clearly desirable to employ a controlled computation scheme to obtain numerically exact results from LDA+DMFT. The QMC method is such a scheme and was already applied by Lichtenstein and collaborators to calculate the magnetic excitation spectrum of ferromagnetic iron [22] and the photoemission spectra of $\mathrm{Sr}_{2} \mathrm{RuO}_{4}$ 23. In the present paper we report on our implementation of the LDA+DMFT(QMC) technique and the results obtained with it for the photoemission spectra of $\mathrm{La}_{1-x} \mathrm{Sr}_{x} \mathrm{TiO}_{3}$.

\section{Computational scheme}

\subsection{Local density approximation (LDA)}

The main problem in combining LDA and model Hamiltonian approaches comes from the fact that their foundations are very different. The LDA employs a functional of the electron density, while Hubbard and Anderson models are formulated in terms of localized, site-centered, atomiclike orbitals. In order to merge the approaches it is necessary to write LDA equations in the basis of such orbitals. There is one variant of the LDA method, based on tight-binding linearized muffin-tin orbitals in the orthogonal approximation (TBLMTO) [24], which is naturally realized in such a basis. The corresponding Hamiltonian can be written as

$$
\begin{aligned}
& H_{\mathrm{LDA}}=\sum_{i l m, j l^{\prime} m^{\prime}, \sigma}\left(\delta_{i l m, j l^{\prime} m^{\prime}} \varepsilon_{i l m} \hat{n}_{i l m}^{\sigma}\right. \\
& \left.+t_{i l m, j l^{\prime} m^{\prime}} \hat{c}_{i l m}^{\sigma \dagger} \hat{c}_{j l^{\prime} m^{\prime}}^{\sigma}\right),
\end{aligned}
$$

where $i, j$ are site indices, $l, m, l^{\prime}, m^{\prime}$ are orbital indices, $\sigma$ is a spin index, and operators carry a hat.

Taking this expression as the non-interacting part of a multi-band periodic Anderson model, one may complement it by a correlation term describing the local contributions to the Coulomb interaction

$$
H_{\text {corr }}=\frac{1}{2} \sum_{i l, m \sigma m^{\prime} \sigma^{\prime}}^{\prime} U_{m m^{\prime}}^{i l} \hat{n}_{i l m \sigma} \hat{n}_{i l m^{\prime} \sigma^{\prime}}
$$

The prime on the sum indicates that at least two of the indices on different operators have to be different. Here, $U_{m m^{\prime}}^{i l}$ denotes the direct Coulomb integral. The much smaller exchange integral and other local contributions of the Coulomb interaction have been neglected. Furthermore, non-local Coulomb contributions are not considered in $H_{\text {corr }}$. Note, that the largest non-local contributions is the nearestneighbor density-density interaction which, to leading order in $Z$ ( $Z$ : number of nearest-neighbor sites), yields only the Hartree term [25] which is already taken into account in the LDA. In an actual calculation, it is not possible to include the local two-particle interaction terms between all orbitals appearing in (2) since the number of states grows exponentially with increasing orbital quantum number. Thus, one usually concentrates on a certain subset of correlations and treats the influence of the remaining states via the use of effective, screened interaction parameters for the shells under consideration. In this spirit, we will assume in the following that it is only necessary to take the Coulomb interaction for the $d$-shell of the transition metal ions $\left(i=i_{d}\right.$ and $\left.l=l_{d}\right)$ explicitly into account; therefore the indices $i l$ will be omitted. The correlation part of the Hamiltonian then acts only on the $d$-wave functions of the transition metal ions. All other valence orbitals will be treated as bands of itinerant electrons which are well described by the LDA.

One must take into account, however, that the Coulomb interaction is already present in LDA in some averaged way. Hence, to avoid double-counting one needs to subtract this term from the LDA-Hamiltonian. Unfortunately, there exists no direct microscopic or diagrammatic link between the Hubbard model approach and LDA, and it is thus not possible to express the LDA energy rigorously via the $d-d$ Coulomb interaction parameter $U$ (except for the atomic limit where one can make a connection between the Coulomb parameter $U$ of the Hubbard model and the second derivative of the atomic total energy as a function of the number of electrons). While it is known that LDA eigenvalues are rather bad approximations for excitation energies of systems with strong Coulomb interactions, the LDA total energy as a function of the number of electrons is a much better approximation for the exact functional. Furthermore, the values of $U$ obtained from LDA calculations often agree well with experimental data and more rigorous calculations.

Therefore one may expect that the LDA part of the Coulomb interaction energy is well approximated by the averaged value of the Coulomb interaction energy in (2)

$$
E_{\text {corr }}:=\frac{1}{2} U n_{d}\left(n_{d}-1\right)
$$


Here, $U$ is the mean value of the Coulomb interaction and may be obtained from a first-principles constrained LDA calculation [26] or from experiment, e.g. high-energy spectroscopy; $n_{d}$ is the total number of $d$-electrons.

In LDA, one-electron energies are defined as derivatives of the total energy as a function of the occupation numbers for the corresponding states. Hence, the oneelectron energy level for the non-interacting d-states is obtained by [15]

$$
\varepsilon_{d}^{0}:=\frac{d}{d n_{d}}\left(E_{\mathrm{LDA}}-E_{\mathrm{corr}}\right)=\varepsilon_{d}^{\mathrm{LDA}}-U\left(n_{d}-\frac{1}{2}\right)
$$

with $\varepsilon_{d}^{\mathrm{LDA}}:=\frac{d}{d n_{d}} E_{\mathrm{LDA}}$, and $E_{\mathrm{LDA}}$ being the total energy calculated from $H_{\mathrm{LDA}}$.

Then, the new non-interacting Hamiltonian will have the form

$$
H_{\mathrm{LDA}}^{0}=\sum_{i l m, j l^{\prime} m^{\prime}, \sigma}\left(\delta_{i l m, j l^{\prime} m^{\prime}} \varepsilon_{i l m}^{0} \hat{n}_{i l m}^{\sigma}+t_{i l m, j l^{\prime} m^{\prime}} \hat{c}_{i l m}^{\sigma \dagger} \hat{c}_{j l^{\prime} m^{\prime}}^{\sigma}\right),
$$

where $\varepsilon_{i l m}^{0}=\varepsilon_{i l m}\left(1-\delta_{l, l_{d}}\right)+\varepsilon_{d}^{0} \delta_{l, l_{d}}$. In reciprocal space, the matrix elements of the operator $H_{\mathrm{LDA}}^{0}$ are given by:

$$
\begin{aligned}
\left(H_{\mathrm{LDA}}^{0}(\mathbf{k})\right)_{q l m, q^{\prime} l^{\prime} m^{\prime}}= & \left(H_{\mathrm{LDA}}(\mathbf{k})\right)_{q l m, q^{\prime} l^{\prime} m^{\prime}} \\
& -\delta_{q l m, q^{\prime} l^{\prime} m^{\prime}} \delta_{q l, q_{d} l_{d}} U\left(n_{d}-\frac{1}{2}\right) .
\end{aligned}
$$

Here $q$ is an index of the atom in the elementary unit cell, $\left(H_{\mathrm{LDA}}(\mathbf{k})\right)_{q l m, q^{\prime} l^{\prime} m^{\prime}}$ is the matrix element in $\mathrm{k}$-space of $H_{\mathrm{LDA}}$, and $q_{d}$ denotes the d-atoms in the unit cell. This non-interacting part $H_{\mathrm{LDA}}^{0}$, taken together with the interaction part (2),

$$
H=H_{\mathrm{LDA}}^{0}+H_{\mathrm{corr}}
$$

forms the $a b$ initio Hamiltonian $H$ for a particular material under investigation.

\subsection{Dynamical mean-field theory (DMFT)}

In general, the investigation of the correlated-electron Hamiltonian $\mathcal{H}$ is too complicated to allow for an exact solution or even a numerical investigation with more than about 10 sites. Here, the DMFT [4, 5, 6] is a powerful approximation scheme which takes into account electronic correlations and, in particular, correctly describes the formation of a coherent quasiparticle band and incoherent Hubbard bands.

The DMFT maps the lattice problem onto a singlesite problem, which is equivalent to a multi-band singleimpurity Anderson model, with the self-consistency condition [15] (the $k$-integrated Dyson equation)

$$
\begin{aligned}
G_{q l m, q^{\prime} l^{\prime} m^{\prime}}(z)=\int \frac{\mathrm{d} \mathbf{k}}{V_{B}} & {\left[z \delta_{q l m, q^{\prime} l^{\prime} m^{\prime}}-\left(H_{\mathrm{LDA}}^{0}(\mathbf{k})\right)_{q l m, q^{\prime} l^{\prime} m^{\prime}}\right.} \\
& \left.-\delta_{q l, q_{d} l_{d}} \Sigma_{q l m, q^{\prime} l^{\prime} m^{\prime}}(z)\right]^{-1}
\end{aligned}
$$

Here, $[\ldots]^{-1}$ implies the inversion of the matrix with elements $n(=q l m), n^{\prime}\left(=q^{\prime} l^{\prime} m^{\prime}\right)$, and integration extends over the Brillouin zone with volume $V_{B}$. In the present study we consider a cubic-crystal structure and assume the $t_{2 g}$ orbitals to be interacting. Due to the high symmetry of the crystal, these three $t_{2 g}$ orbitals are degenerate. Without symmetry breaking, the Green function and the self-energy remain degenerate, i.e., $G_{q l m, q^{\prime} l^{\prime} m^{\prime}}(z)=$ $G(z) \delta_{q l m, q^{\prime} l^{\prime} m^{\prime}}$ and $\Sigma_{q l m, q^{\prime} l^{\prime} m^{\prime}}(z)=\Sigma(z) \delta_{q l m, q^{\prime} l^{\prime} m^{\prime}}$ for $l=$ $l_{d}$ and $q=q_{d}$ (where $l_{d}$ and $q_{d}$ denote the Ti $t_{2 g}$ orbitals). If the partially filled band under consideration is well separated from other bands, as in the case of $\mathrm{La}_{1-x} \mathrm{Sr}_{x} \mathrm{TiO}_{3}$ and other transition metal oxides, one can describe the physics of the partially filled band at the Fermi energy approximately by an effective three band Hamiltonian $H_{\text {LDA }}^{0 \text { eff }}$, e.g., by downfolding to a basis with $t_{2 g}$ orbitals only. One obtains (indices $l=l_{d}$ and $q=q_{d}$ suppressed):

$$
G_{m m^{\prime}}(z)=\int \frac{\mathrm{d} \mathbf{k}}{V_{B}}\left[(z-\Sigma(z)) \delta_{m, m^{\prime}}-\left(H_{\mathrm{LDA}}^{0 \text { eff }}(\mathbf{k})\right)_{m, m^{\prime}}\right]^{-1} \text {. ( }
$$

Due to the diagonal structure of the self-energy the interacting Green function can be expressed via the noninteracting Green function $G^{0}(z)$ :

$$
G(z)=G^{0}(z-\Sigma(z))=\int d \omega \frac{\rho^{0}(\omega)}{z-\Sigma(z)-\omega},
$$

Thus, it is possible to use the Hilbert transformation of the unperturbed LDA-calculated density of states $\rho^{0}(\omega)$ and we do so in the following. This approximation is justified if the hybridization between the $t_{2 g}$ orbitals and the other orbitals is rather weak as in the case of $\mathrm{LaTiO}_{3}$.

The DMFT single-site problem depends on $\mathcal{G}^{-1}=$ $G^{-1}+\Sigma$ and can be formulated in terms of Grassmann variables $\psi$ and $\psi^{*}$. For the local Green function at a Matsubara frequency $\omega_{\nu}=(2 \nu+1) \pi / \beta$, orbital index $m$, and spin $\sigma$ one obtains:

$$
G_{\nu m}^{\sigma}=-\frac{1}{\mathcal{Z}} \int \mathcal{D}[\psi] \mathcal{D}\left[\psi^{*}\right] \psi_{\nu m}^{\sigma} \psi_{\nu m}^{\sigma *} e^{\mathcal{A}\left[\psi, \psi^{*}, \mathcal{G}^{-1}\right]},
$$

where the single-site action $\mathcal{A}$ is given by

$$
\begin{aligned}
& \mathcal{A}\left[\psi, \psi^{*}, \mathcal{G}^{-1}\right]=\sum_{\nu, \sigma, m} \psi_{\nu m}^{\sigma *}\left(\mathcal{G}_{\nu m}^{\sigma}\right)^{-1} \psi_{\nu m}^{\sigma} \\
& -\frac{U}{2} \sum_{(m \sigma) \neq\left(m \sigma^{\prime}\right)_{0}} \int_{0}^{\beta} d \tau \psi_{m}^{\sigma *}(\tau) \psi_{m}^{\sigma}(\tau) \psi_{m^{\prime}}^{\sigma^{\prime}}(\tau) \psi_{m^{\prime}}^{\sigma^{\prime}}(\tau) \cdot(1)
\end{aligned}
$$

\subsection{Quantum Monte-Carlo method (QMC)}

To solve the effective single-site problem defined above we will apply the QMC method which allows for a numerically exact solution 12. To this end, the imaginary time is first discretized into $\Lambda$ steps of size $\Delta \tau\left(\Delta \tau=0.25 \mathrm{eV}^{-1}\right.$ throughout this work), and $\tau_{t}:=t \Delta \tau$ with integer $t$. Then, in a second step, the Hubbard-Stratonovich transformation

$$
\begin{aligned}
& \exp \left\{\frac{\Delta \tau U}{2}\left(\psi_{m t}^{\sigma *} \psi_{m t}^{\sigma}-\psi_{m^{\prime} t}^{\sigma^{\prime} *} \psi_{m^{\prime} t}^{\sigma^{\prime}}\right)^{2}\right\}= \\
& \frac{1}{2} \sum_{s_{m \sigma ; m^{\prime} \sigma^{\prime}}^{t}= \pm 1} \exp \left\{\lambda s_{m \sigma ; m^{\prime} \sigma^{\prime}}^{t}\left(\psi_{m t}^{\sigma *} \psi_{m t}^{\sigma}-\psi_{m^{\prime} t}^{\sigma^{\prime} *} \psi_{m^{\prime} t}^{\sigma^{\prime}}\right)\right\}(12)
\end{aligned}
$$


is employed, which replaces the interacting system by a sum of $2^{\left(2 M^{2}-M\right) \Lambda}$ different non-interacting systems, where $M$ is the number of interacting orbitals. Each non-interacting system can be solved by a Gauss integration which yields a contribution $\left(\mathbf{M}^{\mathbf{s}}\right)^{-1} \operatorname{det}\left(\mathbf{M}^{\mathbf{s}}\right)$ to the Green function in imaginary time, parameterized by $\left(t, t^{\prime}\right)$, where

$$
\begin{aligned}
{\left[\mathbf{M}_{m}^{\sigma \mathbf{s}}\right]_{t t^{\prime}}=} & \Delta \tau^{2}\left[\left(\mathbf{G}_{m}^{\sigma}\right)^{-1}+\boldsymbol{\Sigma}_{m}^{\sigma}\right]_{t t^{\prime}} \\
& -\delta_{t t^{\prime}} \sum_{m^{\prime} \sigma^{\prime}} \lambda_{m \sigma ; m^{\prime} \sigma^{\prime}} \tilde{\sigma}_{m \sigma ; m^{\prime} \sigma^{\prime}} s_{m \sigma ; m^{\prime} \sigma^{\prime}}^{t}
\end{aligned}
$$

Here, $\tilde{\sigma}_{m \sigma ; m^{\prime} \sigma^{\prime}}=2 \Theta\left(\sigma^{\prime}-\sigma+\delta_{\sigma \sigma^{\prime}}\left[m^{\prime}-m\right]\right)-1$ has a different sign if $(m \sigma)$ and $\left(m^{\prime} \sigma^{\prime}\right)$ are exchanged. Since a full summation over all non-interacting systems is computationally impossible, the Monte-Carlo method is employed for importance sampling. Details of the one-band QMC algorithm in the context of DMFT can be found in Refs. 12. [6] and for the band-degenerate case in Refs. [27].

\section{Results and Discussion}

The stoichiometric compound $\mathrm{LaTiO}_{3}$ has a perovskite crystal structure with a small orthorhombic distortion $\left(\angle T i-O-T i \approx 155^{\circ}\right)$ 28]. Below $T_{N}=125 \mathrm{~K}$ [29] it is an antiferromagnetic insulator [30] with a Ti magnetic moment of $0.45 \mu_{B}$ and a small energy gap of approximately $0.2 \mathrm{eV}$ [31, 32. At doping $x=0.05, \mathrm{La}_{1-x} \mathrm{Sr}_{x} \mathrm{TiO}_{3}$ undergoes an insulator-to-metal transition and becomes a correlated paramagnetic metal with a strongly enhanced susceptibility and electronic specific heat coefficient [33].

The standard LDA calculation for undoped $\mathrm{LaTiO}_{3}$ yields a density of states (Fig. 11) which is typical for early transition metal oxides with a completely filled $2 p$ oxygen band ranging from $-8.2 \mathrm{eV}$ to $-4.0 \mathrm{eV}$ and a partially filled Ti-3 $d$ band above it. Since the Ti-ion has an octahedral coordination of oxygen ions, the Ti-3d band is split into three degenerate $t_{2 g}$ and two degenerate $e_{g}$ subbands which do not mix. Titanium is three-valent in $\mathrm{LaTiO}_{3}$ and the corresponding formal ionic configuration is $d^{1}$. This implies a partially filled $t_{2 g}$ subband containing one electron with a total capacity of six electrons. The $e_{g}$ subband is empty and situated just above the $t_{2 g}$ subband. In order to simplify our calculations the real orthorhombic crystal structure was replaced by a cubic structure with the same volume. This approximation leads to a slight overestimation of the effective bandwidth.

The $t_{2 g}$ band is the only partially filled band and is well separated from other bands. Therefore in our DMFT calculation we took only Coulomb interactions between electrons in $t_{2 g}$ orbitals into account. The resulting problem is equivalent to a Hubbard model with three degenerate bands. The DMFT chemical potential was adjusted to yield the doping $x=0.06$, i.e. 0.94 electrons in the $t_{2 g}$ band. We employ Eq. (3) within constrained LDA [26], i.e., changing the number of $t_{2 g}$-electrons only, to calculate $U$. Our LMTO-ASA calculation (TB-LMTO-ASA code of Andersen and coworkers [24] version 47) yields a fully-screened Coulomb interaction of $U=4.2 \mathrm{eV}$ within

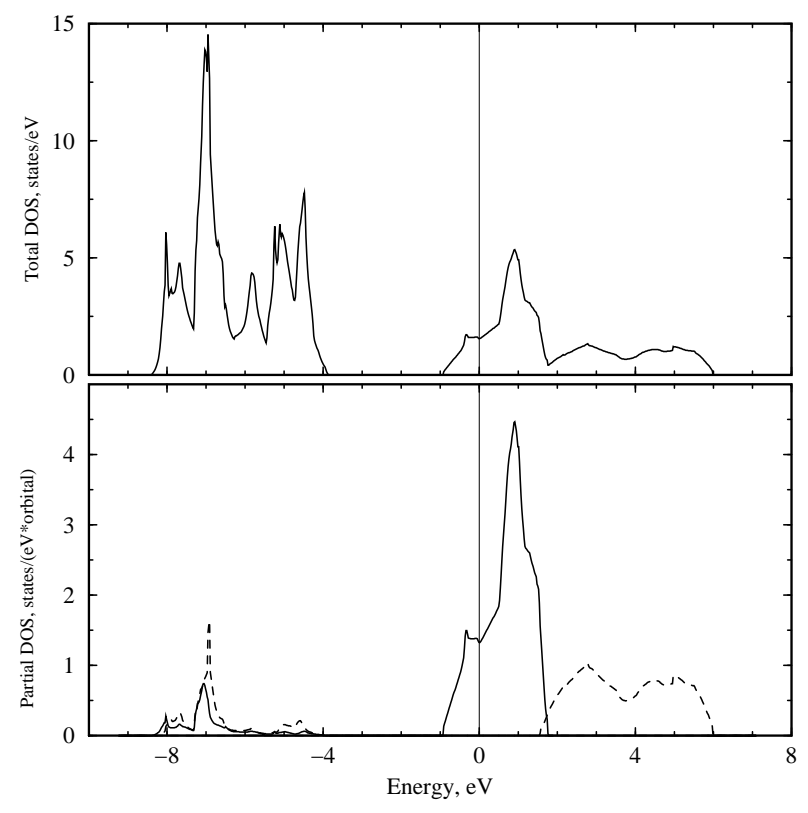

Fig. 1. Densities of states of $\mathrm{LaTiO}_{3}$ calculated with LDALMTO. Upper figure: total density of states; lower figure: partial $t_{2 g}$ (solid lines) and $e_{g}$ (dashed lines) densities of states.

the basis $\operatorname{Ti}(4 \mathrm{~s}, 4 \mathrm{p}, 3 \mathrm{~d}) \mathrm{La}(6 \mathrm{~s}, 6 \mathrm{p}, 5 \mathrm{~d}) \mathrm{O}(2 \mathrm{~s}, 2 \mathrm{p})$ at a Wigner Seitz radius of 2.37 a.u. for Ti. Our result has to be compared to that of Solovyev et al. [34] who obtained $U=3.2$ $\mathrm{eV}$ employing the ASA-LMTO method within orthogonal representation. This shows that the ab-initio calculation of $U$, which is the interaction between particular $\left(t_{2 g}\right)$ orbitals, is rather sensitive to the orthogonality of the wave functions and, also, to the choice of the orbitals. Unless specified otherwise, we will, thus, use $U=4 \mathrm{eV}$ and keep in mind that the inherent uncertainty is about $0.5 \mathrm{eV}$.

In Fig. 2, the spectral function obtained from our $\mathrm{LDA}+\mathrm{DMFT}(\mathrm{QMC})$ calculation at temperature $T \approx 1000 \mathrm{~K}$ is compared with the non-interacting $t_{2 g}$ density of states. One can see the typical features of the spectra of strongly correlated systems: a lower Hubbard band, a well pronounced quasi-particle peak, and an upper Hubbard band. While for the non-interacting case $100 \%$ of the spectral weight is located in the quasi-particle band, the LDA+DMFT spectra are characterized by a spectral weight transfer from the quasiparticle band to the Hubbard bands and a narrowing of the quasiparticle band.

The QMC simulations performed in this paper to solve the effective multi-band single-impurity model provide a numerically exact solution, but require a large computational effort which restricted our calculations to temperatures of the order of $T=0.1 \mathrm{eV}(\approx 1000 \mathrm{~K})$. Since this technique yields results only at imaginary (Matsubara-) frequencies the calculation of the spectral function requires an analytic continuation of the spectral function via, e.g., the maximum entropy method [35]. Previous LDA+DMFT investigations of the photoemission spectra of $\mathrm{La}_{1-x} \mathrm{Sr}_{x} \mathrm{TiO}_{3}$ used a variant of IPT for doped multi-band systems [15] and NCA 20] to solve the DMFT-equations, i.e., approximate techniques. In Fig. 3, 


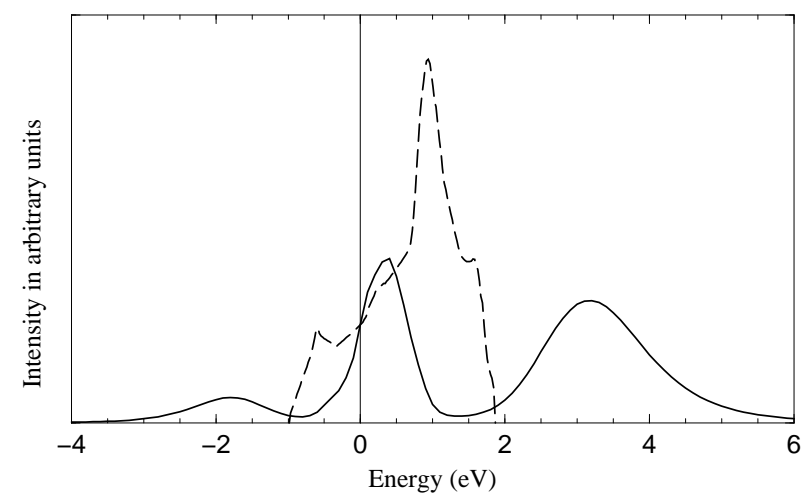

Fig. 2. Partial $t_{2 g}$ densities of states of $\mathrm{LaTiO}_{3}$ calculated with LDA+DMFT(QMC) (solid lines) and LDA (dashed lines).

we compare the results obtained within these approximations with the numerically exact QMC simulation, all at $T \approx 1000 \mathrm{~K}$. One notes that within IPT the shape of the upper Hubbard band is not correct. Moreover, there is no quasiparticle peak at $1000 \mathrm{~K}$, the reason being that IPT underestimates the Kondo temperature considerably such that the very narrow quasiparticle peak found at low temperatures (see right inset of Fig. 3) disappears already at about $250 \mathrm{~K}$. A similarly narrow IPT quasiparticle peak was found in a three-band model study with BetheDOS by Kajueter and Kotliar 36]. While NCA comes off much better than IPT it still underestimates the width of the quasiparticle peak by a factor of two. Furthermore, the position of the quasiparticle peak is too close to the lower Hubbard band. In the left inset of Fig. 3, the behavior at the Fermi level is shown. At the Fermi level, the NCA yields a spectral function which is almost by a factor two too small. The shortcomings of the NCAresults appear to result from the well-known problems which this approximation scheme encounters already in the single-impurity Anderson model at low temperatures and/or low frequencies 37, 38. Similarly, the deficiencies of the IPT-results are not entirely surprising in view of the semi-phenomenological nature of this approximation, especially for a system off half filling. This comparison shows that the choice of the method used to solve the DMFT equation is indeed important.

Photoemission spectroscopy of the early transition meta oxides provides a direct tool for the study of the electronic structure of strongly correlated materials. A comparison of the experimental photoemission spectra 40 with results obtained from LDA and LDA+DMFT(QMC) at $1000 \mathrm{~K}$ 41 are shown in Fig. 4. To take into account the uncertainty in $U$, we present results for $U=3.2,4.25$ and $5 \mathrm{eV}$. All spectra are multiplied with the Fermi step function and Gaussian-broadened with a broadening parameter of $0.3 \mathrm{eV}$ to simulate the experimental resolution 40 . The LDA band structure calculation clearly fails to reproduce the broad band observed in the experiment at 1-2 eV below the Fermi energy [40]. Taking the correlations between the electrons into account, this lower band is easily identified as the lower Hubbard band whose spectral weight originates from the quasiparticle band at the

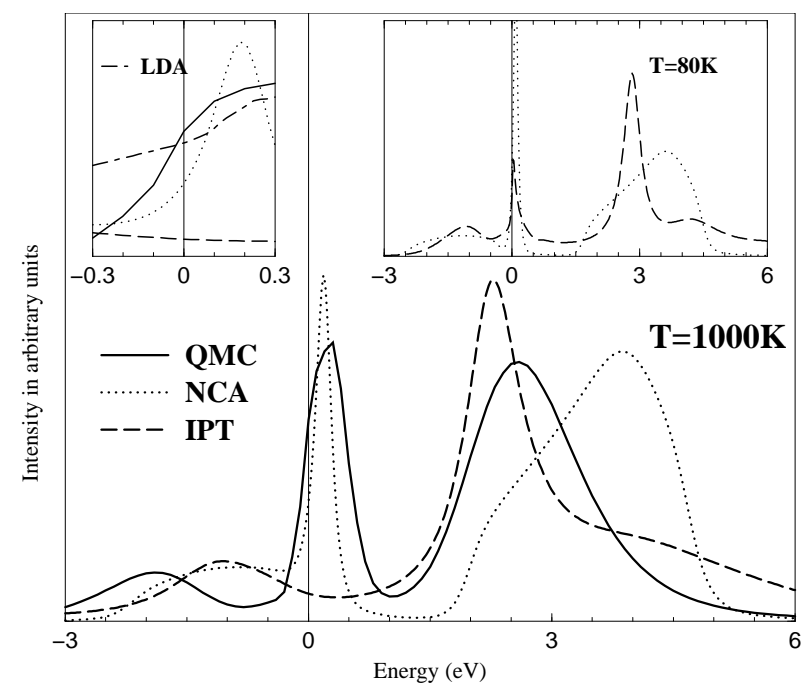

Fig. 3. Comparison of the spectral densities of $\mathrm{La}_{1-x} \mathrm{Sr}_{x} \mathrm{TiO}_{3}$ $(x=0.06)$ as calculated by LDA+DMFT using the approximations IPT and NCA, with the numerically exact QMC-result at $T=0.1 \mathrm{eV}$, i.e., approximately $1000 \mathrm{~K}$, and $U=4 \mathrm{eV}$. Inset left: Behavior at the Fermi level including the LDA DOS. Inset right: NCA and IPT spectra for the temperature $80 \mathrm{~K}$.

Fermi energy and increases with $U$. The best agreement with experiment concerning the relative intensities of the Hubbard band and the quasi-particle peak and, also, the position of the Hubbard band is found for $U=5 \mathrm{eV}$ 43. The value $U=5 \mathrm{eV}$ is still compatible with the abinitio calculation of this parameter. One should also note that the photoemission experiment is sensitive to surface properties. Due to the reduced coordination number at the surface, the bandwidth is likely to be smaller and the Coulomb interaction to be less screened, i.e., larger. Both effects make the system more correlated and, thus, might also explain why better agreement is found for $U=5 \mathrm{eV}$. Besides, the polycrystalline nature of the sample and, also, spin and orbital 44 fluctuation, not taken into account in the LDA+DMFT approach, could further reduce the quasiparticle weight.

In conclusion, the $\mathrm{LDA}+\mathrm{DMFT}(\mathrm{QMC})$ approach is shown to be a workable computational scheme which merges the conventional band structure approach with a recently developed many-body technique in combination with a numerically reliable evaluation method. Thereby, it provides a powerful tool for future $a b$ initio investigations of real materials with strong electronic correlations. The LDA+DMFT(QMC) approach not only explains the existence of the lower Hubbard band in doped $\mathrm{LaTiO}_{3}$, but also, in contrast to LDA, reproduces the qualitative picture of the spectral weight transfer from the quasiparticle band to the lower Hubbard band, the position of the lower Hubbard band, and the narrowing of the quasiparticle band. 


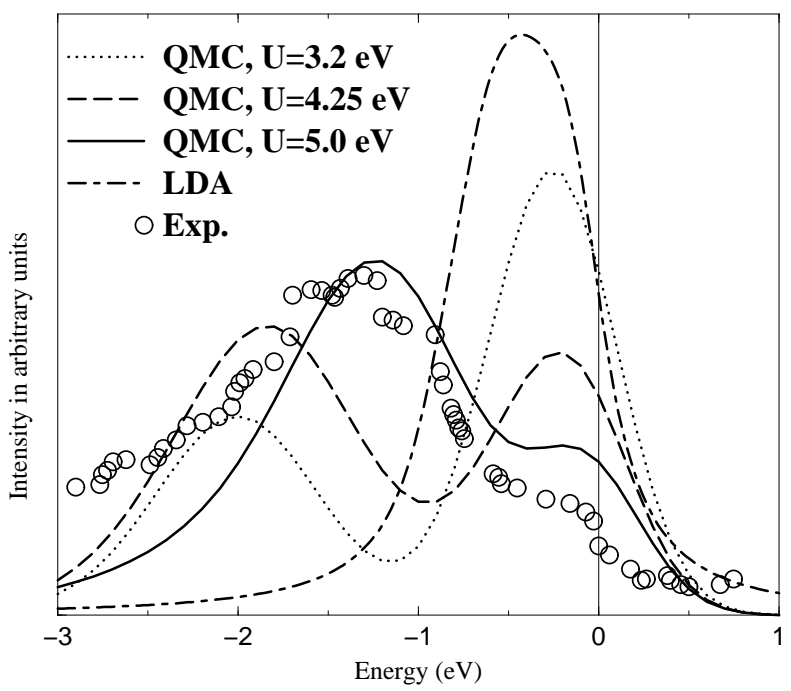

Fig. 4. Comparison of the experimental photoemission spectrum [40], the LDA result, and the LDA+DMFT(QMC) calculation for $\mathrm{LaTiO}_{3}$ with $6 \%$ hole doping and different Coulomb interaction $U=3.2,4.25$, and $5 \mathrm{eV}$.

\section{Acknowledgment}

We are grateful to R. Claessen, J. Lægsgaard, Th. Pruschke, G. A. Sawatzky, J. Schmalian, and M. Zölfl for useful discussions. This work was supported in part by the Sonderforschungsbereich 484 of the Deutsche Forschungsgemeinschaft and the Russian Foundation for Basic Research (RFFI-98-02-17275).

\section{References}

1. P. Hohenberg and W. Kohn, Phys. Rev. B 136, 864 (1964); W. Kohn and L. J. Sham, Phys. Rev. 140, 4A, A1133 (1965).

2. R. O. Jones and O. Gunnarsson, Rev. Mod. Phys. 61, 689 (1989).

3. V. I. Anisimov, J. Zaanen, and O. K. Andersen, Phys. Rev. B 44, 943 (1991); V. I. Anisimov, F. Aryasetiawan, and A. I. Lichtenstein, J. Phys. Cond. Matter 9, 767 (1997).

4. D. Vollhardt in Correlated Electron Systems, edited by V. J. Emery, World Scientific, Singapore, 1993, p. 57.

5. Th. Pruschke, M. Jarrell, and J. K. Freericks, Adv. in Phys. 44, 187 (1995).

6. A. Georges, G. Kotliar, W. Krauth, and M. J. Rozenberg, Rev. Mod. Phys. 68, 13 (1996).

7. W. Metzner and D. Vollhardt, Phys. Rev. Lett. 62, 324 (1989).

8. A. Georges and G. Kotliar, Phys. Rev. B 45, 6479 (1992).

9. H. Keiter and J. C. Kimball, Phys. Rev. Lett. 25, 672 (1970); N. E. Bickers, D. L. Cox, and J. W. Wilkins, Phys. Rev. B 36, 2036 (1987).

10. Th. Pruschke and N. Grewe, Z. Phys. B 74, 439 (1989).

11. Th. Pruschke, D. L. Cox, and M. Jarrell, Phys. Rev. B 47, 3553 (1993).

12. J. E. Hirsch and R. M. Fye, Phys. Rev. Lett. 56, 2521 (1986); M. Jarrell, Phys. Rev. Lett. 69, 168 (1992); M.
Rozenberg, X. Y. Zhang, and G. Kotliar, Phys. Rev. Lett. 69, 1236 (1992); A. Georges and W. Krauth, Phys. Rev. Lett. 69, 1240 (1992).

13. M. Caffarel and W. Krauth, Phys. Rev. Lett. 72, 1545 (1994).

14. R. Bulla, to appear in Adv. Sol. State Phys. (2000), preprint cond-mat/0003377.

15. V. I. Anisimov, A. I. Poteryaev, M. A. Korotin, A. O. Anokhin, and G. Kotliar, J. Phys. Cond. Matter 9, 7359 (1997).

16. A. I. Lichtenstein and M. I. Katsnelson, Phys. Rev. B 57, 6884 (1998).

17. V. Drchal, V. Janiš, and J. Kudrnovský in Electron Correlations and Material Properties, edited by A. Gonis, N. Kioussis, and M. Ciftan, Kluwer/Plenum, New York 1999, p. 273.

18. J. Lægsgaard and A. Svane, Phys. Rev. B 58, 12817 (1998).

19. Th. Wolenski, Combining bandstructure and dynamical mean-field theory: A new perspective on $\mathrm{V}_{2} \mathrm{O}_{3}$, $\mathrm{PhD}$ Thesis, Universität Hamburg 1998, Shaker Verlag, Aachen 1999.

20. M. B. Zölfl, Th. Pruschke, J. Keller, A. I. Poteryaev, I. A. Nekrasov, and V. I. Anisimov, Phys. Rev. B 61, 12810 (2000).

21. M. I. Katsnelson and A. I. Lichtenstein, J. Phys. Cond. Matter 11, 1037 (1999).

22. M. I. Katsnelson and A. I. Lichtenstein, preprint condmat/9904428 (1999).

23. A. Liebsch and A. Lichtenstein, Phys. Rev. Lett. 84, 1591 (2000).

24. O. K. Andersen, Phys. Rev. B 12, 3060 (1975); O. Gunnarsson, O. Jepsen, and O. K. Andersen, Phys. Rev. B 27, 7144 (1983).

25. E. Müller-Hartmann, Z. Phys. B 74, 507 (1989); J. Wahle et al. Phys. Rev. B 58, 12749 (1998).

26. O. Gunnarson, O. K. Andersen, O. Jepsen, and J. Zaanen, Phys. Rev. B 39, 1708 (1989).

27. M. J. Rozenberg, Phys. Rev. B 55, R4855 (1997); J. E. Han, M. Jarrell, and D. L. Cox, Phys. Rev. B 58, R4199 (1998); K. Held and D. Vollhardt, Euro. Phys. J. B 5, 473 (1998).

28. D. A. MacLean, H.-N. Ng, and J. E. Greedan, J. Solid State Chem. 30, 35 (1979).

29. J. P. Gopel, J. E. Greedan, and D. A. MacLean, J. Solid State Chem. 43, 244 (1981).

30. M. Eitel and J. E. Greedan, Journal of the Less-Common Metals 116, 95 (1986).

31. Y. Okimoto, T. Katsufuji, Y. Okada, T. Arima, and Y. Tokura, Phys. Rev. B 51, 9581 (1995).

32. J. P. Goral, J. E. Greedan, and D. A. MacLean, J. Solid State Chem. 43, 244 (1982).

33. Y. Tokura, Y. Taguchi, Y. Okada, Y. Fujishima, T. Arima, K. Kumagai, and Y. Iye, Phys. Rev. Lett. 70, 2126 (1993).

34. I. V. Solovyev, N. Hamada, and K. Terakura, Phys. Rev. B 53, 7158 (1996).

35. For a review on the maximum entropy method see M. Jarrell and J. E. Gubernatis, Physics Reports 269, 133 (1996). Here, the code of A. Sandvik was employed. Note, that the maximum entropy method is most reliable in the vicinity of the Fermi energy. At larger energies, the energy resolution becomes poorer.

36. H. Kajueter and G. Kotliar, Int. J. Mod. Phys. 11, 729 (1997). 
37. E. Müller-Hartmann, Z. Phys. B 57, 281 (1984).

38. NCA is a self-consistent perturbation theory for the singleimpurity Anderson model and is often a fast method to obtain results for this model and also within DMFT. However, NCA is known to violate Fermi liquid properties at temperatures much lower than the smallest energy scale of the problem and whenever charge excitations become important [37, 39]. Hence, in some parameter ranges it fails to account for the proper low-energy physics and must therefore be applied with considerable care [39].

39. Th. Pruschke, D. L. Cox, and M. Jarrell, Phys. Rev. B 47, 3553 (1993).

40. A. Fujimori et al, Phys. Rev. Lett. 69, 1796 (1992). A. Fujimori et al, Phys. Rev. B 46, 9841 (1992). A qualitatively and quantitatively similar spectrum was obtained recently by Yosida et al., preprint cond-mat/9911446, with an experimental energy resolution of only $30 \mathrm{meV}$. One may suppose, however, that broadening effects due to the polycrystalline nature of the sample and surface effects are, then, larger than the instrumental resolution.

41. At present, QMC simulations of the DMFT equations are not feasible at the experimental temperature $(80 \mathrm{~K})$. We note, however, that no intrinsic temperature dependence was observed in the experiment 40, at least up to room temperature.

42. In Fig. 1, the distance between the lower Hubbard band and the chemical potential is seen to decrease with increasing $U$. Note, however, that the upper Hubbard band is also shifted to higher energies.

43. If one multiplies the IPT and NCA results of Fig. 3 with the Fermi function and employs a broadening of $0.3 \mathrm{eV}$ one obtains a spectrum where the lower Hubbard band is nearer to the Fermi energy and which has a less pronounced minimum or shoulder between Hubbard and quasi-particle band.

44. B. Keimer et al., preprint cond-mat/0002014. 